\title{
DE
}

DE GRUYTER

OPEN

BULGARIAN ACADEMY OF SCIENCES

CYBERNETICS AND INFORMATION TECHNOLOGIES • Volume 14, Special Issue

Sofia $\bullet 2014$

Print ISSN: 1311-9702; Online ISSN: 1314-4081

DOI: $10.2478 /$ cait-2014-0043

\section{IoT Forest Environmental Factors Collection Platform Based on ZIGBEE}

\author{
Zhang Yu, Liu Xugang, Geng Xue, Li Dan
}

School of Information and Computer Engineering, Northeast Forestry University, Harbin, CO 150040 China

Email: ld@nefu.edu.cn

\begin{abstract}
Nowadays the development of Internet of Things (IoT) technology has witnessed great changes in the world. As it has often been mentioned, IoT Environment Monitoring Technologies and IOT Smart Home Technologies have been gradually accepted by people and have good prospects for development. Now we can research a networking based intelligent platform to monitor our forest environmental factors in time with the new IoT technology based on ZIGBEE protocol. ZIGBEE based networking technologies has the advantages of low power dissipation, low data rate and high-capacity transportation, which makes it more suitable for the design of the node of forest environmental factors collection platform. So, we are going to discuss a kind of IoT forest environment factors collection platform based on ZIGBEE protocol.
\end{abstract}

Keywords: ZIGBEE, Internet of Things (IoT), Forest Environmental Factors Collection Platform (FEFCP).

\section{Introduction}

Wireless Sensor Networks (WSN) have been developed in the past few years for static and mobile networks [1]. The nodes in a WSN are self-organized, having the ability to communicate with each other and to collaborate in order to achieve the goals of a network, depending on the application areas related to industry, health monitoring, control and military area [2]. Thanks to recent advances in wireless 
technologies, these WSNs are amenable to support a variety of real-world applications [11], including health care, environment control, intelligent buildings, etc. [12].

As it is well known, there is a close connection between WSN and IoT, whose key technology is still Internet technology. Over the last couple of decades, Internet has been in a constant state of evolution. The early days of Internet were characterized by the World Wide Web (WWW) [14], however, nowadays with the appearance of IoT, the definition of Internet is becoming more generalized. Hightech hardware and software infrastructures, such as air transportation systems, bullet trains and Internet, make humans and any objects interacting with each other more and more closely and tightly [3], and there is no exception in the relation between us and the forest environmental factors. Forest Environmental Collection Platform is essential to monitor our forest environmental factors.

\section{Internet of things}

\subsection{Summarization of IoT}

Kevin Ashton firstly proposed the concept of IoT in 1999, and he referred to IoT as uniquely identifiable interoperable connected objects with Radio Frequency IDentification (RFID) technology. However, the exact definition of IoT is still in a forming process that is subject to the perspectives taken [4].

IoT is an important part of a new generation of information technologies. This has two meanings: the first is that the core and foundation of IoT is still Internet, the Internet based on the extension and expansion of the network. The second is that the extension and expansion of its clients to any object and the object between the information exchange and communication.

Thus in the definition viewed in this perspective, Internet of Things includes the radio frequency identification, infrared sensors, global positioning systems, laser scanners and other information sensing device, according to an agreed protocol, to any object connected to the Internet, information exchange and communication in order to achieve the object of intelligent identifying, locating, tracking, monitoring and managing a network. Things are second in the world information industry computer, the Internet and mobile communication networks are after the third wave.

\subsection{The IoT principle}

First, it is widely used in a variety of sensing technologies. And we can consider IoT's key technologies in a hardware and software aspect (Fig. 1) [5].

Internet is a massive deployment of various types of sensors, each sensor is a source of information, the different information content and format of the different types of information captured by the sensor. This includes the sensor data obtained in real time and a certain frequency of the periodic collection of the environmental information, or continuously undated data. 


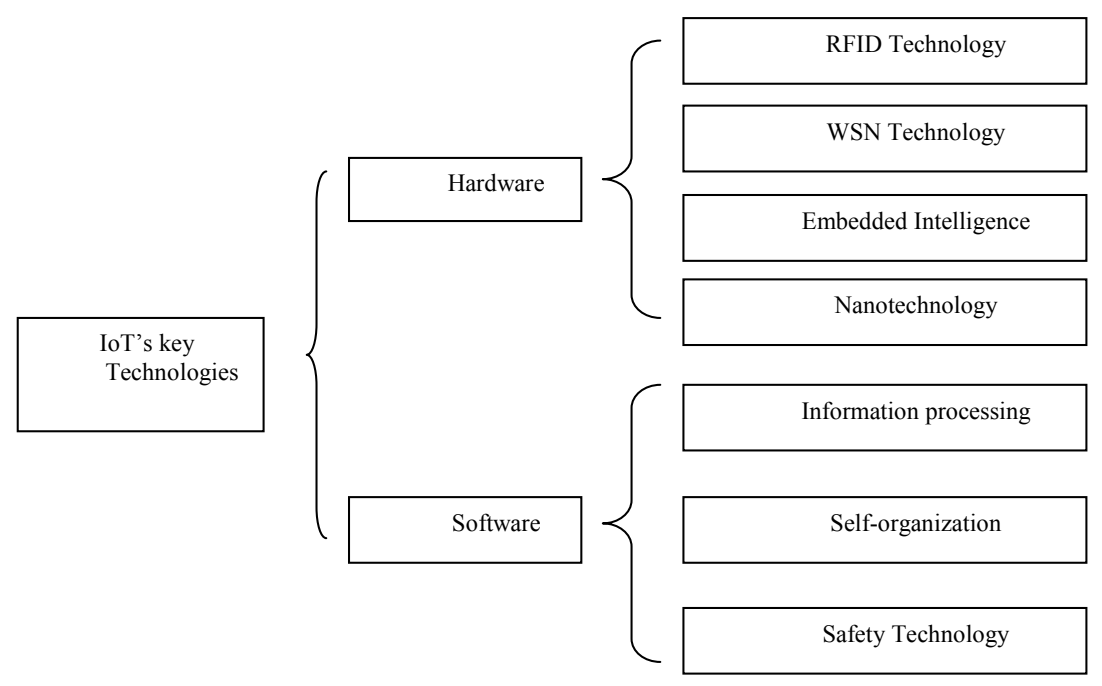

Fig. 1. IoT's key technologies

Secondly, it is a network based on Internet. The important foundation and core networking technology is still Internet; through a variety of wired and wireless networks and Internet integration, the information will pass in real time objects accurately. Things timing sensors collect online information transmitted over the network in need. Because of its extremely large number and the requirement to guarantee the accuracy and time, forming a mass of information in the transmission process must be adapted to a variety of heterogeneous networks and protocols.

Besides, things not only provide the connection of the sensor, which has the ability to intelligent processing of objects that can implement intelligent control. Things will be a combination of sensors and intelligent processing, the use of cloud computing, pattern recognition and other smart technologies, expanding its field of application. Analyzing vast amounts of information obtained from the sensors, processing and handling of meaningful data in order to meet the different needs of different users, discover new applications and application modes.

In short, it is an aggregation of Things applications and technology after the modern IT development to a certain stage of the upgrade, various sensing technologies, modern network technology and artificial intelligence aggregation, and integration and automation technology applications, the wisdom of the dialogue and objects create wisdom of the world.

IoT technology extend from three-in-one network technology, whose key technology is achieved eventually by IP of the three networks. The joint point of the three-in-one network is the optimized network based on IP [6-8]. As we can see from the Fig. 2, it is the IoT's architecture and protocol suite. We have divided our IoT system into four layers using the hierarchy thought, which can make our whole system much easier to complete. 


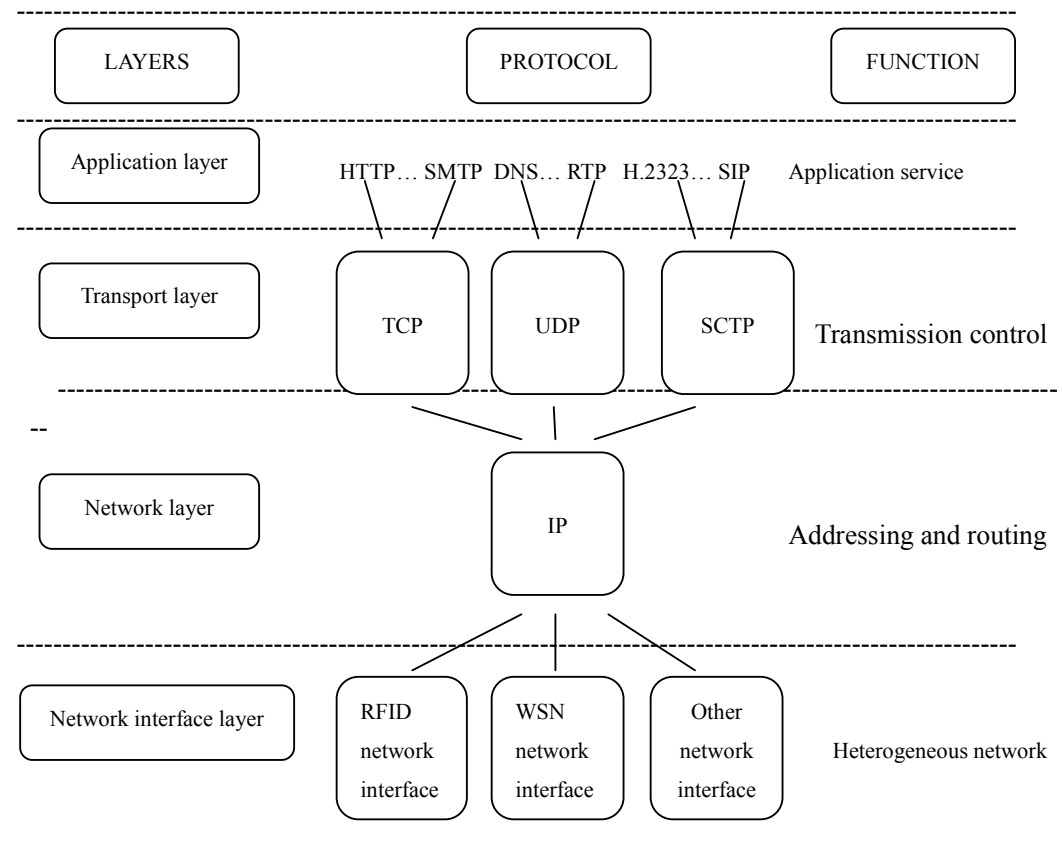

Fig. 2. IoT's architecture and protocol suite

\subsection{The history of IoT}

IoT's concept is proposed in 1999, its definition is simple: put all the articles and other information via radio frequency identification and sensing devices to connect things together, for intelligent identification and management. In other words, things refer to various types of sensors and the existing mutual convergence of a new Internet technology.

The developed in 2004 conception by the Japanese Ministry of Internal Affairs proposed u-Japan, and it is intended to be built in Japan in 2010 as an "Anytime, Anywhere, Anything, Anyone" can access environment. In the same year the South Korean government developed the u-Korea strategy, Han Guoxin pass issued by the Ministry, "the digital age humanism: IT839 strategy" to specific echoes u-Korea.

In November, 2005 in Tunis World Summit of the Information Society (WSIS), the International Telecommunication Union (ITU) released the "ITU Internet Report 2005: The Internet of Things". The report noted, that the ubiquitous "Internet of Things" communication era is approaching the world, all objects from tires to toothbrushes, from housing to the tissue can actively be exchanged via Internet. RFID, sensor technology, nanotechnology, intelligent embedded technology must be more widely used. IBM in November 2008 stated that "the wisdom of the Earth" concept, namely the "Internet + Internet of Things = Wisdom of the Earth", uses this as a strategy for economic revitalization. If the execution of the infrastructure, the implantation of "wisdom" concept, not only in a short term can be a powerful economic stimulus and promote employment, but also it can cause China to build a mature wisdom infrastructure platform in a short time. 
In June 2009, when the European Commission proposed an action plan for the Internet of Things, and clearly expressed that the technical aspects of the program will be given sufficient financial support, the government raised the level of the existing laws and regulations to adapt to network monitoring solutions.

In August 2009, when Premier Wen Jiabao visited Wuxi Industrial Development sensor network, he explicitly instructed the early planning for the future, early break-core technology, and specifically called for the establishment of China's sensor information center, as soon as possible, called "Experience China" center.

\subsection{The composition and role of the IoT}

IoT appropriate formation starts from the receiver, CPU, data transmission path, storage, operating systems, applications, data transmission and so on. In the past the idea has been limited to physical infrastructure and IT infrastructure apart: on one hand, this is the airport and other physical buildings, but on the other hand it is the data center. In the era of things, these physical facilities and network infrastructure will be perfectly integrated. At the same time things can be applied to our daily life, such as real-time monitoring of the physical condition of the elderly, perfect combination of own the household appliances. IoT would be an opportunity for the information age, but also a challenge.

\section{ZIGBEE technology}

\subsection{ZIGBEE}

The ZIGBEE Alliance developed a global standard designed for WSN which required scalability, a low cost, a high reliability and a low data rate [9], which we call ZIGBEE protocol. ZIGBEE technology is a kind of wireless connection technology, which can work under radio frequency of $2.4 \mathrm{GHz}$ (Global Pop), 868 $\mathrm{MHz}$ (Euro-Pop) and $915 \mathrm{MHz}$ (American Pop). Its transmission distance is between $10 \mathrm{~cm}$ up to $75 \mathrm{~cm}$, which can also be increased if needed. As a kind of wireless communication technology, ZIGBEE has the advantages of low power consumption, low complexity, low rate of compound, inexpensive and short distance transmission, which can mainly be used in the data transmission devices that are short-haul, with low power consumption and low transmission rate. And it can also be applied to periodical and intermittent data transmission.

In brief, ZIGBEE is a highly reliable wireless data transmission net, whose transmission distance is within the range from the standard $75 \mathrm{~m}$ up to hundred meters, a few kilometers and even infinity. ZIGBEE are wireless data communication networks which are made of 65000 wireless data transmitting modules. Across the whole ZIGBEE network, every wireless data transmitting module can communicate with each other. And every node transmission distance can be extended from $75 \mathrm{~m}$ up to infinity. 


\subsection{Communication theory}

ZIGBEE protocol is a low distance wireless technology standard developed by ZIGBEE Alliance. This protocol uses IEEE802.15.4, which was originally designed for personal area networks [12], as it is PHY layer and MAC layer protocol. So the device follows ZIGBEE protocol, it also follows IEEE802.15.4 standard. ZIGBEE protocol stack is shown in Fig. 3. The ZIGBEE stack has a layered structure which contains four different layers with the two upper layers being the application and network layers, while the lower layers have been defined by IEEE 802.15.4 standard, as MAC and PHY layers [10].

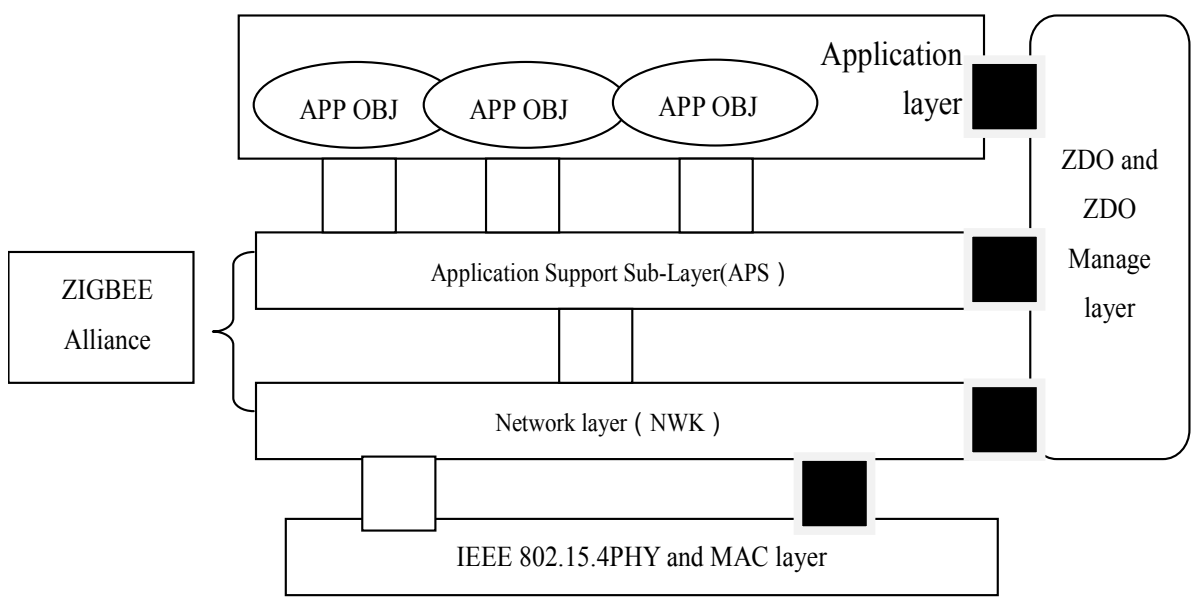

Fig. 3. The structural schematic diagram of ZIGBEE protocol

At the MAC layer, ZIGBEE mainly uses CSMA-CA of 802.11 series standard to improve the compatibility of the system.

In the aspect of a network, ZIGBEE enables three network topologies (Fig. 4): a star; a mesh and a cluster-tree [13].
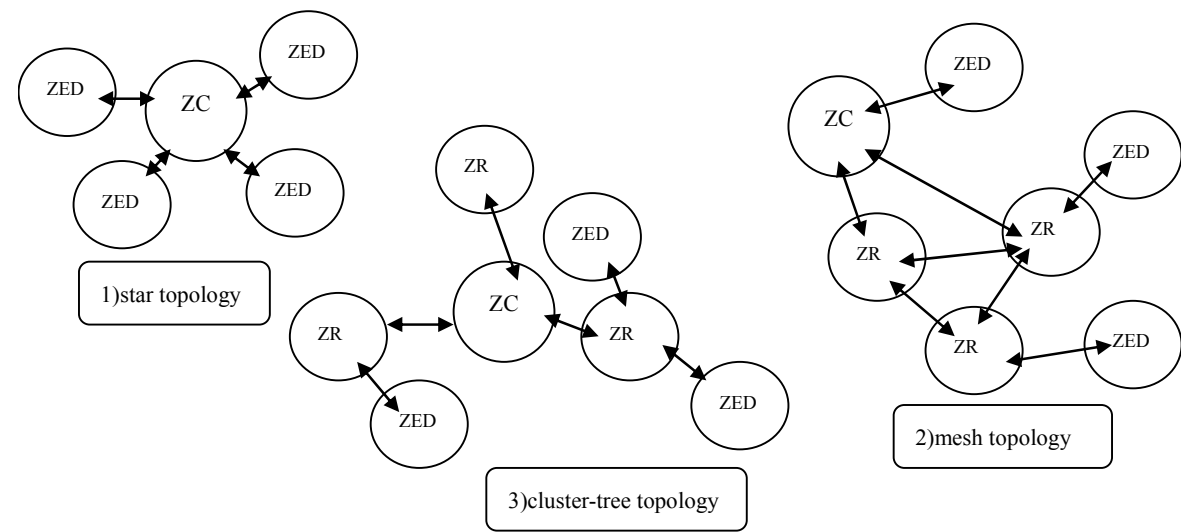

Fig. 4. Network topologies 
In star topology (Fig. 4-1), the communication paradigm is centralized, while the communication paradigm of the mesh topology (Fig. 4-2) is decentralized. The cluster-tree topology (Fig. 4-3) is a special case of the mesh network where there is a single routing path between any pair of nodes [13].

We can divide the data collection layer (the sensing layer of IoT system) into three layers. They are the data collection sensor layer, ZIGBEE node layer and ZIGBEE coordinator layer. We call it three layers structure of the data collection layer here (Fig. 5).

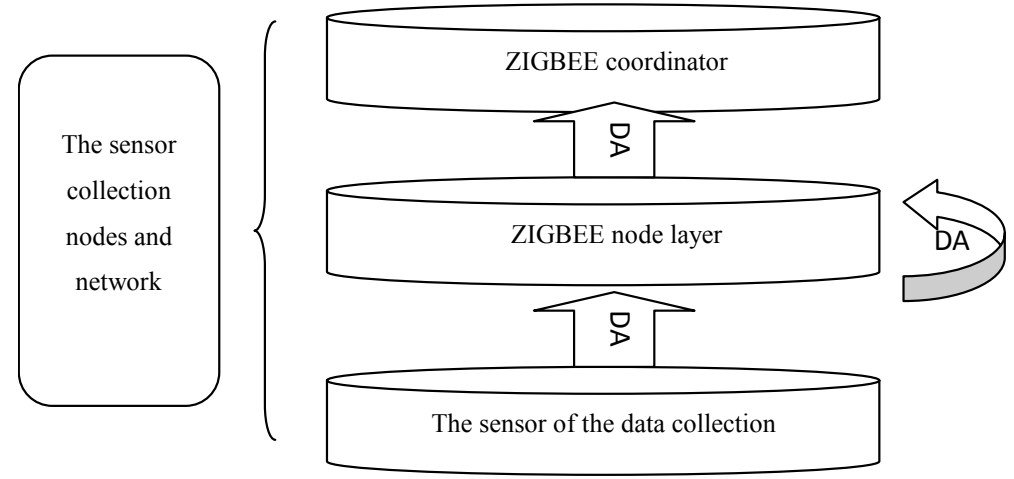

Fig. 5. The three layers structure of the data collection layer

Fig. 4 is the theoretical structure of the data collection layer. Then, according to this theoretical figure, we can achieve the following structure - the practical structure (Fig. 6).

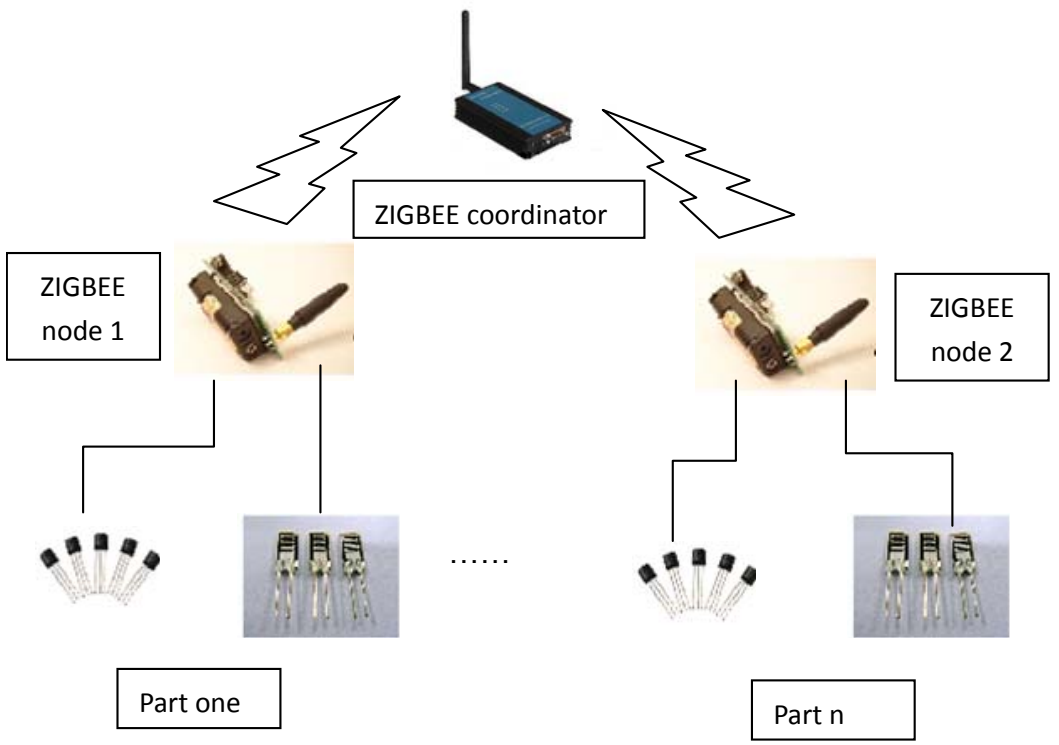

Fig. 6. The practical structure of the data collection layer 


\section{The design of FEFCP}

\subsection{Summarization}

As it has been above mentioned, FEFCP is a collection platform which is designed to gain access to the timely monitoring of the forest environmental factors by using IoT technology and computer technology, so that we can realize the information of monitoring of the forest environmental factors.

\subsection{The aim of FEFCP}

Our study is aimed at solving the shortcomings of the traditional monitor platforms. As we know, WSN consists of a set of nodes that can interact with their environment by observing and controlling the physical phenomena, such as vibration, humidity and pressure [12]. Obviously, we can use this kind of IoT to monitor the forest environmental factors, which we call FEFCP. Our FEFCP is designed to monitor the forest environmental factors timely, so that we can control the factors accurately and on time. Besides, this platform has a user-friendly interface, so that we can acknowledge the information collected by our platform easily.

\subsection{The design of the nodes information}

As we all know, the data of the IoT owns a time-space relativity feature. And the IoT sensor nodes are somewhat different from the common ones. Since the data collected by our forest environmental factors collection nodes has a spatial attribute and a time attribute the geographic locations of every sensor nodes (longitude and latitude), and the acquisition time of information. That is to say, the platform designed by us must also adjust to these two features of the IoT data. So we should timely monitor our forest environmental factors according to the geographic locations (Fig. 7).

As it can be seen from the above figure, we can know the information of every node in detail. Our FEFCP system is just like a satellite map - when we move the mouse to a location on the map, we can thoroughly get this place in forest environmental factors information, which makes the data we collected easily visible. 


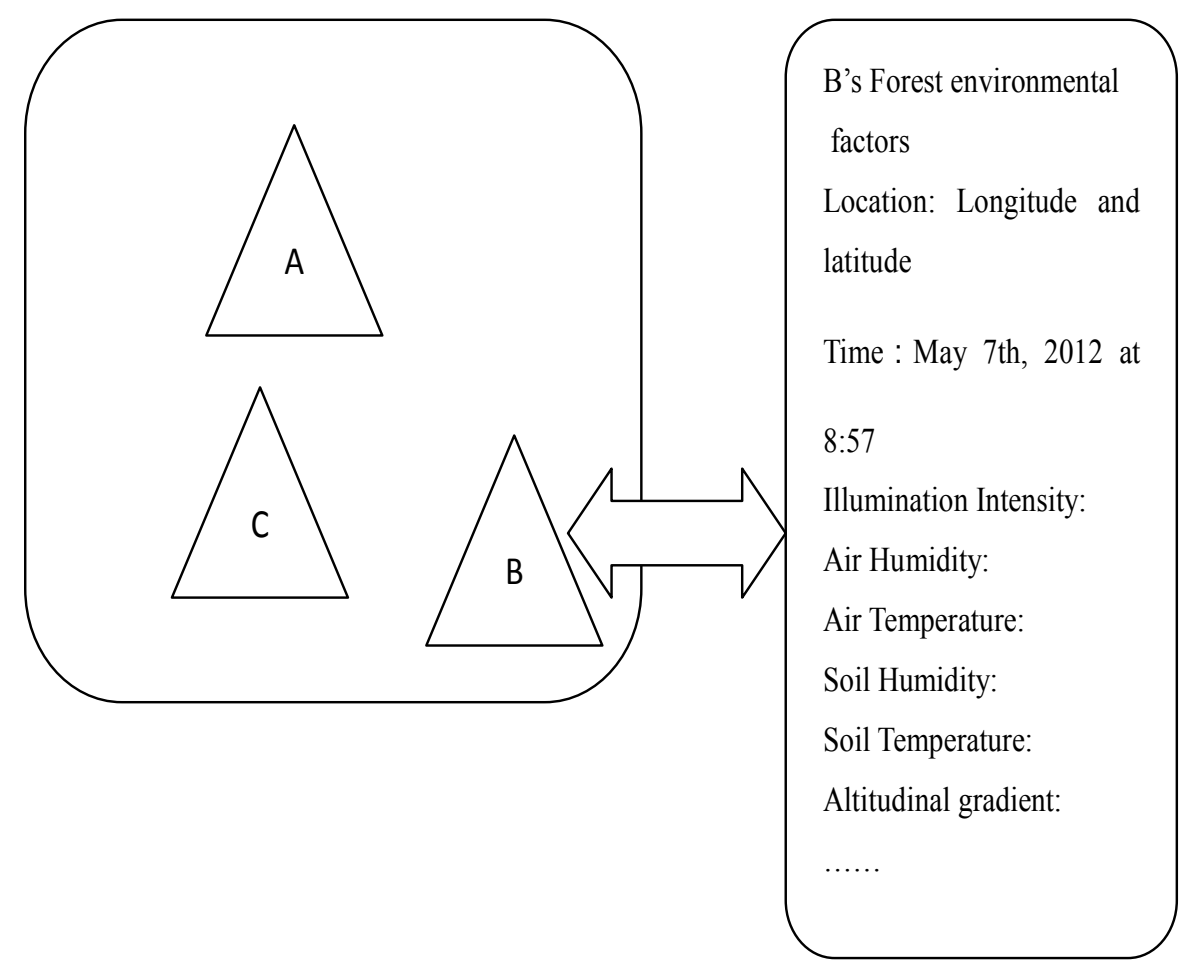

Fig. 7. Forest environmental factors collection node

\subsection{The realization of FEFCP}

\subsubsection{The thought of delamination}

The idea of delamination's significance comes from a simple scientific principle, which we call a layering principle [15].

Using this notion has many advantages. Firstly, layering can make our system separable and every layer independent on the other one. Secondly, the systems built through this thought has great flexibility. Last, but not least, using the idea of delamination lets our FEFCP divisible in structure which makes our system easy to realize and maintain.

Considering the above, we will build our FEFCP using the notion of delamination.

\subsubsection{Five subsystems of FEFCP}

Through careful analysis and relevant demand analysis, the author has divided FEFCP into five subsystems (Fig. 8) using delamination. 


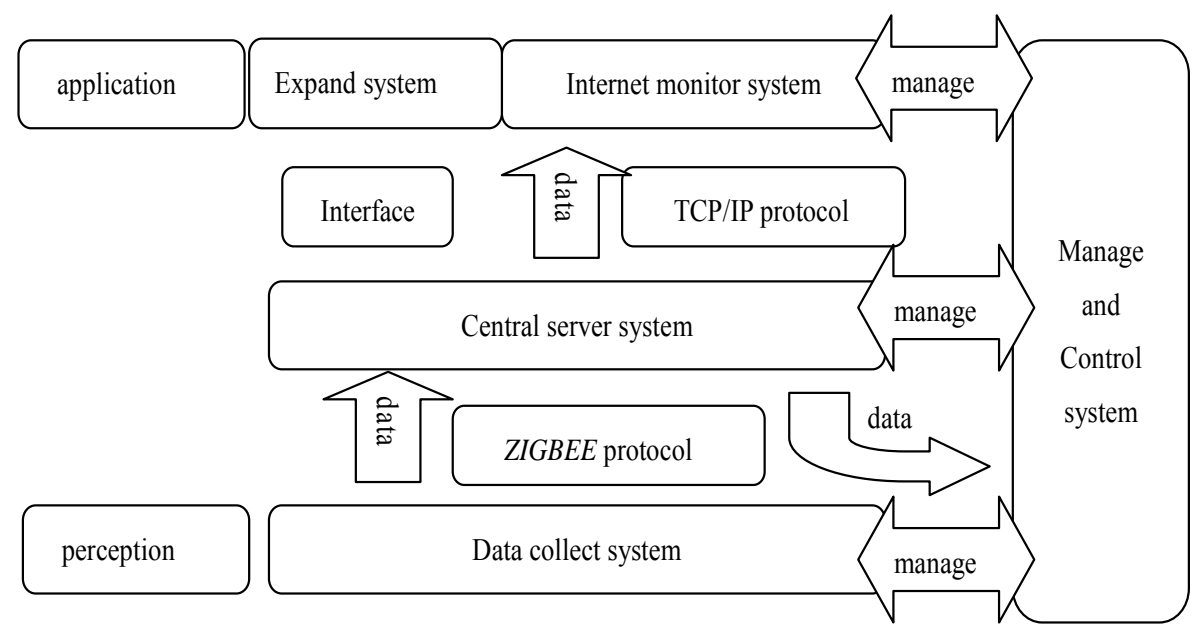

Fig. 8. Forest environmental factors collection platform

As we can see from Fig. 8, our FEFCP consists of five subsystems. They are: Data Collect Subsystem (DCS), Central Server Subsystem (CSS), Manage and Control Subsystem (MCS), Internet Monitor Subsystem (IMS) and EXpand Subsystem (EXS). Then we will introduce these five subsystems in detail.

Firstly, let us talk about our Data Collect Subsystem. As we can see from Fig. 2, the three layers construction of the data collection is exact what we framed it as a Data Collect System. To put it simply, based on ZIGBEE protocol, our DCS needs to collect the forest environmental factors timely and then transmit the information collected to our CSS. It is no exaggeration to say that this subsystem is the foundation of the whole FEFCP system.

As for CSS, it is the central part of FEFCP aimed at coordinating the overall system. Obviously, CSS is the kernel system, just like the brain of the whole body. This system is designed to save and analyze the information sent from DCS, as well as transmit the data to the IMS through Internet, based on TCP/IP protocol. Meanwhile, our CSS also needs to send the analysis results of the data to MCS so that we can achieve informing and intelligent monitoring of the forest environmental factors.

More specifically, this Central Server Subsystem proposes to use SQLSERVER database to save the data collected by DCS.

Then IMS will be realized by using relevant web technology.

Fourthly, MCS has been divided into two parts - forest environmental factors control and system model control.

It is easy for us to understand the control of the forest environmental factors, which means analyzing the data sent from CSS for intelligent adjustment and control of the forest environmental factors. As for the model control, we can simply comprehend that there are three models - a standard model, a power saving model and a brainpower model (there can be various kinds of models to adapt many different scenes). As we all know, ZIGBEE technology is famous for its power saving, in fact, however, some ZIGBEE sensor nodes can consume two AA 
batteries for two up to four days (every battery contains $800 \mathrm{mAh}$ ) if we do nothing at all to control the power consumption. Thus we need to save electricity by using different models.

For instance, if it is a rainy day, we can diminish the monitoring rate of the temperature sensor because the probability for a fire disaster is low. And we can also reduce the monitor frequency of illumination intensity sensor if it is at middle night to cut down the consumption, as the change of the illumination intensity is quite low then.

EXS is the expanding subsystem for the whole system. We have considered the third-party application software which needs to access the data in our FEFCP. So, there are some interfaces of functions we provide in our CSS in order our information to be used by the third-party application software. Then we propose the Forest Environmental Factors Collection Platform (FEFCP).

\section{Conclusion}

Aimed to solve the shortcomings of the traditional collection of the forest environmental factors, this paper has put forward a new kind of intelligent FEFCP, which has the advantages of low consumption, good speed and easy visuality. And it is more fit for monitoring of the forest environmental factors.

Acknowledgment: This work has been supported by the Fundamental Research Funds for the Central Universities No 2572014CB24 and China Undergraduate Scientific and Technological Innovation No 201410225031.

\section{References}

1. K a r 1, H., A. W i 11 i g. Protocols and Architectures for Wireless Sensor Networks. London, Wiley, 2005.

2. A l-Harbawi, M., M. Rasid, N. No ordin. Utilizing Neighbours-Table to Improve Tree Touting Protocol in ZigBee Network. - Wireless Pers. Commun., Vol. 65, 2012, 469-488. doi: 10.1007/s11277-011-0268-9.

3. Li, N. et al. A New Methodology to Support Group Decision-Making for IoT-Based Emergency Response Systems. - Inf. Syst. Front., 2013. doi: 10.1007/s10796-013-9407-z.

4. Li, S., L. Xu, S. Zha o. The Internet of Things: A Survey. - Inf. Syst. Front., 2014. doi: 10.1007/s10796-014-9492-7.

5. Qian, Z., Y. Wang. IoT Technology and Application. - Acta Electronica Sinica, 2012. doi:10.3969/j.issn.0372-2112.2012.05.026.

6. L u o, J. et al. Wireless Sensor Network Interconnection Design Based on IPv6 Protocol. - In: 5th International Conference on Wireless Communications, 2009.

7. S o n g, H., et al. 6LoWPAN-Based Tactical Wireless Sensor Network Architecture for Remote Large-Scale Random Deployment Scenarios. - In: IEEE Military Communications Conference, 2009.

8. Jung, W., et al. SSL-Based Lightweight Security of IP-Based Wireless Sensor Networks. In: Intemational Conference on Advanced Information Networking and Applications Workshop, 2009.

9. Specification, Z. ZigBee Document 053474r17. Release r17. Zigbee Alliance. Vol. 17. 2008. 
10. IEEE Standard for Information Technology. Telecommunications and Information Exchange between Systems - Local and Metropolitan Area Networks Specific Requirements. Part 15.4: Wireless Medium Access Control (MAC) and PHysical Layer (PHY) Specifications for Low-Rate Wireless Personal Area Networks (LR-WPANs). IEEE Std 802.15.4-2003, $0 \_1-670$.

11. K a r 1, H., A. W i 11 ig. Protocols and Architectures for Wireless Sensor Networks. New York, Wiley, 2005.

12. O u n i, S., Z. A y o u b. Cooperative Association/Re-association Approaches to Optimize Energy Consumption for Real-Time - IEEE 802.15.4/ZigBee Wireless Sensor Networks. - Wireless Pers. Commun., 2013. doi: 10.1007/s11277-013-0996-0.

13. Cunha, A., M. Tovar. TDBS: A Time Division Beacon Scheduling Mechanism for ZigBee Cluster-Tree Wireless Sensor Networks. - Real-Time Syst., 2008. doi: 10.1007/s11241-0089063-4.

14. T s a i, C., C. L a i, A. V a s i 1 a k o s. Future Internet of Things: Open Issues and Challenges. Wireless Netw., 2014. doi: 10.1007/s11276-014-0731-0.

15. Zhou, J., Z. Y ang. Computer Network Protocol and Layering. - In: Data of Culture and Education, 2006. 